\title{
RAS mutation prevalence among patients with metastatic colorectal cancer: a meta-analysis of real-world data
}

\author{
George Kafatos*,1, Daniela Niepel2 ${ }^{2}$ Kimberley Lowe ${ }^{3}$, Sophie Jenkins-Anderson ${ }^{4}$, Hal \\ Westhead ${ }^{4}$, Tamer Garawin ${ }^{3}$, Zuzana Traugottová ${ }^{5}$, Antonios Bilalis ${ }^{6}$, Edit Molnar ${ }^{7}$, Jozsef \\ Timar $^{8}$, Erika Toth ${ }^{9}$, Nikolaos Gouvas ${ }^{10}$, George Papaxoinis ${ }^{11}$, Samuel Murray ${ }^{12}$, Nadia \\ Mokhtar ${ }^{13}$, Hana Vosmikova ${ }^{14}$, Pavel Fabian ${ }^{15}$, Alena Skalova ${ }^{16}$, Piotr Wójcik ${ }^{17}$, Andrzej \\ Tysarowski $^{18}$, Mario Barugel ${ }^{19}$, J Han van Krieken ${ }^{20}$ \& Jörg Trojan ${ }^{21}$ \\ ${ }^{1}$ Amgen Ltd, Uxbridge, UK \\ ${ }^{2}$ Amgen $\mathrm{GmbH}$, Vienna, Austria \\ ${ }^{3}$ Amgen Inc., Thousand Oaks, CA, USA \\ ${ }^{4}$ Adelphi Research (Global), Manchester, UK \\ ${ }^{5}$ Amgen s.r.o., Prague, Czech Republic \\ ${ }^{6}$ Amgen Hellas EPE, Athens, Greece \\ ${ }^{7}$ Amgen Kft, Budapest, Hungary \\ ${ }^{8}$ Semmelweis Medical University, Budapest, Hungary \\ ${ }^{9}$ National Institute of Oncology, Budapest, Hungary \\ ${ }^{10}$ Gastrointestinal Cancer Study Group, Heraklion, Crete, Greece \\ ${ }^{11}$ Hellenic Cooperative Oncology Group, University of Athens, Athens, Greece \\ ${ }^{12}$ Biomarker Solutions, London, UK \\ ${ }^{13}$ National Cancer Institute, Cairo University, Cairo, Egypt \\ ${ }^{14}$ University Hospital Hradec Kralove, Hradec Kralove, Czech Republic \\ ${ }^{15}$ Masaryk Memorial Cancer Institute, Brno, Czech Republic \\ ${ }^{16}$ Bioptická laboratoř, Plzeň, Czech Republic \\ ${ }^{17}$ Oncogene Diagnostics Sp.z.o.o., Krakow, Poland \\ ${ }^{18}$ Centrum Onkologii Instytut im. Marii Sklodowskiej Curie, Warsaw, Poland \\ ${ }^{19}$ Tecnofarma, Santiago, Chile \\ ${ }^{20}$ Radboud University Medical Center, Nijmegen, The Netherlands \\ ${ }^{21}$ University Hospital, Frankfurt, Germany \\ * Author for correspondence: Tel.: +44 1895 525615; Fax: +44 1895 525104; gkafatos@amgen.com
}

\begin{abstract}
Aim: A confirmed wild-type RAS tumor status is commonly required for prescribing anti-EGFR treatment for metastatic colorectal cancer. This noninterventional, observational research project estimated RAS mutation prevalence from real-world sources. Materials \& methods: Aggregate RAS mutation data were collected from 12 sources in three regions. Each source was analyzed separately; pooled prevalence estimates were then derived from meta-analyses. Results: The pooled RAS mutation prevalence from 4431 tumor samples tested for RAS mutation status was estimated to be $43.6 \%$ (95\% Cl: $38.8-48.5 \%$ ); ranging from $33.7 \%$ (95\% Cl: $28.4-39.3 \%$ ) to $54.1 \%$ (95\% Cl: $51.7-56.5 \%)$ between sources. Conclusion: The RAS mutation prevalence estimates varied among sources. The reasons for this are not clear and highlight the need for further research.
\end{abstract}

First draft submitted: 8 December 2016; Accepted for publication: 6 June 2017; Published online: 27 July 2017

Keywords: anti-EGFR $\bullet B R A F \bullet K R A S \bullet \mathrm{mCRC} \bullet$ metastatic colorectal cancer $\bullet N R A S \bullet$ prevalence $\bullet$ RAS $\bullet$ real world

Colorectal cancer (CRC) was the third most common malignancy among men and the second most common malignancy among women globally in 2012 [1]. Approximately, 60\% of CRC cases occur in developed regions and incidence rates can vary up to tenfold around the world, with the lowest rates observed in South Central Asia and Africa (excluding Southern Africa) [2]. A quarter of patients with CRC already have metastatic disease at the time of their initial presentation and diagnosis, and almost $50 \%$ will subsequently develop metastases [3]. Once 
Table 1. Data sources included in the analysis.

\begin{tabular}{|c|c|c|c|c|}
\hline Data source & Country & Type of data source & Study period & Number of samples ${ }^{\dagger}$ \\
\hline \multicolumn{5}{|l|}{ Pathology \& clinical data } \\
\hline Argentina & Argentina & Amgen-sponsored study & March 2014 to November 2014 & 565 \\
\hline Czech Republic I & Czech Republic & Pathology center & January 2014 to December 2014 & 100 \\
\hline Greece & Greece & Amgen-sponsored study & December 2013 to August 2014 & 514 \\
\hline Hungary I & Hungary & Pathology center & September 2013 to June 2014 & 150 \\
\hline Hungary II & Hungary & Pathology center & September 2013 to June 2014 & 150 \\
\hline Middle East Registry & $\begin{array}{l}\text { Algeria, Egypt, Saudi Arabia, } \\
\text { UAE, Lebanon, Kuwait, Bahrain }\end{array}$ & $\mathrm{mCRC}$ registry & 2013 to 2014 & 188 \\
\hline \multicolumn{5}{|l|}{ Pathology-only data } \\
\hline Czech Republic II & Czech Republic & Pathology center & January 2014 to December 2014 & 100 \\
\hline Czech Republic III & Czech Republic & Pathology center & January 2014 to December 2014 & 100 \\
\hline Middle East Pathology I & $\begin{array}{l}\text { Algeria, Saudi Arabia, UAE, } \\
\text { Lebanon, Kuwait, Bahrain }\end{array}$ & $\mathrm{mCRC}$ registry & 2012 to 2015 & 447 \\
\hline Middle East Pathology II & Egypt & $\mathrm{mCRC}$ registry & 2012 to 2015 & 303 \\
\hline Poland I & Poland & Pathology center & January 2014 to December 2014 & 1664 \\
\hline Poland II & Poland & Pathology center & January 2014 to December 2014 & 150 \\
\hline
\end{tabular}

metastatic disease has emerged, the 5-year survival rate is approximately 5-15\%, with a median overall survival of 9-30 months [4].

In the past decade, new treatment options have become available to patients with metastatic CRC (mCRC) that include the use of irinotecan- and oxaliplatin-containing chemotherapies, the use of antiangiogenic drugs, such as bevacizumab, and the introduction of monoclonal antibodies (mAbs), such as cetuximab and panitumumab, which target the EGFR [5]. A number of studies have shown that RAS biomarker status (exons 2, 3 and 4 of the KRAS and NRAS oncogenes) is predictive of patients' treatment response to anti-EGFR mAbs specifically, and patients who have $R A S$ wild-type tumors are significantly more likely to benefit from anti-EGFR treatment [6-10]. As a result, treatment guidelines for anti-EGFR therapies recommend that patients with mCRC should have confirmed wild-type $R A S$ tumor status prior to the initiation of treatment [11]. There have been a limited number of studies that have attempted to use real-world data to estimate the prevalence of $R A S$ mutations among patients with mCRC. The majority of data relating to $R A S$ mutation prevalence in $\mathrm{mCRC}$, which have been published previously, are reported from clinical trials.

The primary objective of this research project was to estimate the prevalence of $R A S$ mutations in patients with mCRC using different real-world data sources. These estimates include an overall estimate and estimates calculated according to patients' demographic and clinical characteristics. Additional objectives included estimating the prevalence of non-KRAS exon 2 mutations (KRAS exons 3, 4 and $N R A S$ exons 2, 3, 4) in patients with a confirmed wild-type $K R A S$ exon 2 status and estimating the prevalence of $B R A F$ mutations among patients with $\mathrm{mCRC}$.

\section{Materials \& methods}

\section{Data sources}

This noninterventional, observational research project used aggregate patient data, rather than individual patient data, from multiple real-world data sources to estimate $R A S$ mutation prevalence. Data were collected from 12 data sources (three local mCRC registries, two industry-sponsored studies [Amgen] and seven pathology centers) across 12 countries (Algeria, Argentina, Bahrain, Czech Republic, Egypt, Greece, Hungary, Kuwait, Lebanon, Poland, Saudi Arabia and the United Arab Emirates) (Table 1). Recruitment periods for the observational studies and registries providing the aggregate data spanned a range of different time periods (from 2012 to 2015). The study period for the majority of the data sources was between January and December 2014.

The data were collected using a standardized online data collection form for all samples according to both their $R A S$ mutation status and a number of demographic and clinical variables. In addition, the turnaround times for $R A S$ testing of the samples were also requested. The online data collection form was distributed to each data source, with data entry organized locally. A pilot trial of the online form was conducted to ensure any issues with the 
electronic data collection were addressed before full implementation of the form. Data checks were subsequently carried out after receiving data from each source to determine if there were any errors or missing data that required correction or clarification. Turnaround time was defined as the number of days between the laboratory receiving the request for a $R A S$ test to be performed and the result being reported to the requesting oncologist. Only aggregate data were requested with no possible link to individual patient information.

\section{Patient eligibility criteria}

To be eligible for the study, patients were required to have a recorded diagnosis of $\mathrm{mCRC}$ and a tumor sample that had been tested for $R A S$ mutation status. Some samples were initially tested for $K R A S$ exon 2 status only, with testing for the remaining RAS exons (KRAS exons 3, 4 and NRAS exons 2, 3, 4) conducted at a later time point. In practice, samples were likely to have been collected at different time points (e.g., time of surgery or immediately prior to initiation of anti-EGFR therapy), including some which may have been collected prior to metastatic diagnosis (most likely stage III CRC).

\section{Statistical analysis}

The prevalence of $R A S$ and $B R A F$ mutations (defined generically herein as 'biomarker' $[b m]$ ) was estimated as follows:

\section{$100 \times$ Number of samples with bm mutation}

\section{Number of samples with bm mutation + Number of samples with bm wild type}

The prevalence of $R A S$ and $B R A F$ mutations was estimated from all samples taken from patients with mCRC. The prevalence of non-KRAS exon 2 mutations (i.e., mutations of KRAS exons 3, 4 and NRAS exons 2, 3, 4) was also estimated for all samples from patients with $\mathrm{mCRC}$ and a confirmed wild-type KRAS exon 2 status. The prevalence estimates are reported with their corresponding 95\% CIs. The Clopper-Pearson (exact) method was used for CI calculation. For each data source, prevalence was also estimated according to a number of covariate patient characteristics, including patient age, gender, the site the tumor sample was taken from, the clinical indication for sampling, presence or absence of metastases, tumor staging and line of treatment at the time of testing. Comparison between the prevalence estimates by different variables was carried out based on $95 \%$ CIs and p-values (using Pearson $\chi^{2}$ testing).

The meta-analysis was performed using a random effects model to consolidate the results of the primary outcome analysis, and account for the heterogeneity of the data sources, particularly with respect to variables such as testing methods, time periods and patient characteristics. The Freeman-Tukey double arcsine transformation method was used to stabilize the variance. The Hodges-Lehman estimator - a robust and nonparametric estimator of a population's location parameter - was used for the overall point estimate of prevalence. The meta-analysis was carried out using the 'meta' command in Stata (StataCorp. 2013. Stata Statistical Software: Release 2013, StataCorp LP, TX, USA).

\section{Results}

Aggregate data from 4431 tumor samples from 12 data sources were included in the $R A S$ mutation prevalence analysis. Aggregate data from 2561 tumor samples from six data sources were included in the $B R A F$ analysis. Aggregate data from 2606 tumor samples from 11 data sources were included in the analysis of non-KRAS exon 2 (KRAS exons 3, 4 and NRAS exons 2, 3, 4) samples with KRAS exon 2 wild-type samples. The size of these data sources varied from 100 tumor samples (Czech Republic pathology centers) to 1664 tumor samples (Polish pathology center) (Table 1).

\section{RAS mutation prevalence}

The overall pooled $R A S$ mutation prevalence across the 12 data sources included in the analysis was estimated to be $43.6 \%$ (95\% CI: 38.8-48.5\%). RAS mutation prevalence varied between data sources, ranging from 33.7\% (95\% CI: 28.4-39.3\%) in the Middle Eastern pathology dataset and 34.6\% (95\% CI: 27.7-42.1\%) in the Middle Eastern mCRC registry to $53.6 \%$ (95\% CI: 43.2-63.8\%) and 54.1\% (95\% CI: 51.7-56.5\%) in two European pathology centers, in the Czech Republic and Poland, respectively (Figure 1). 


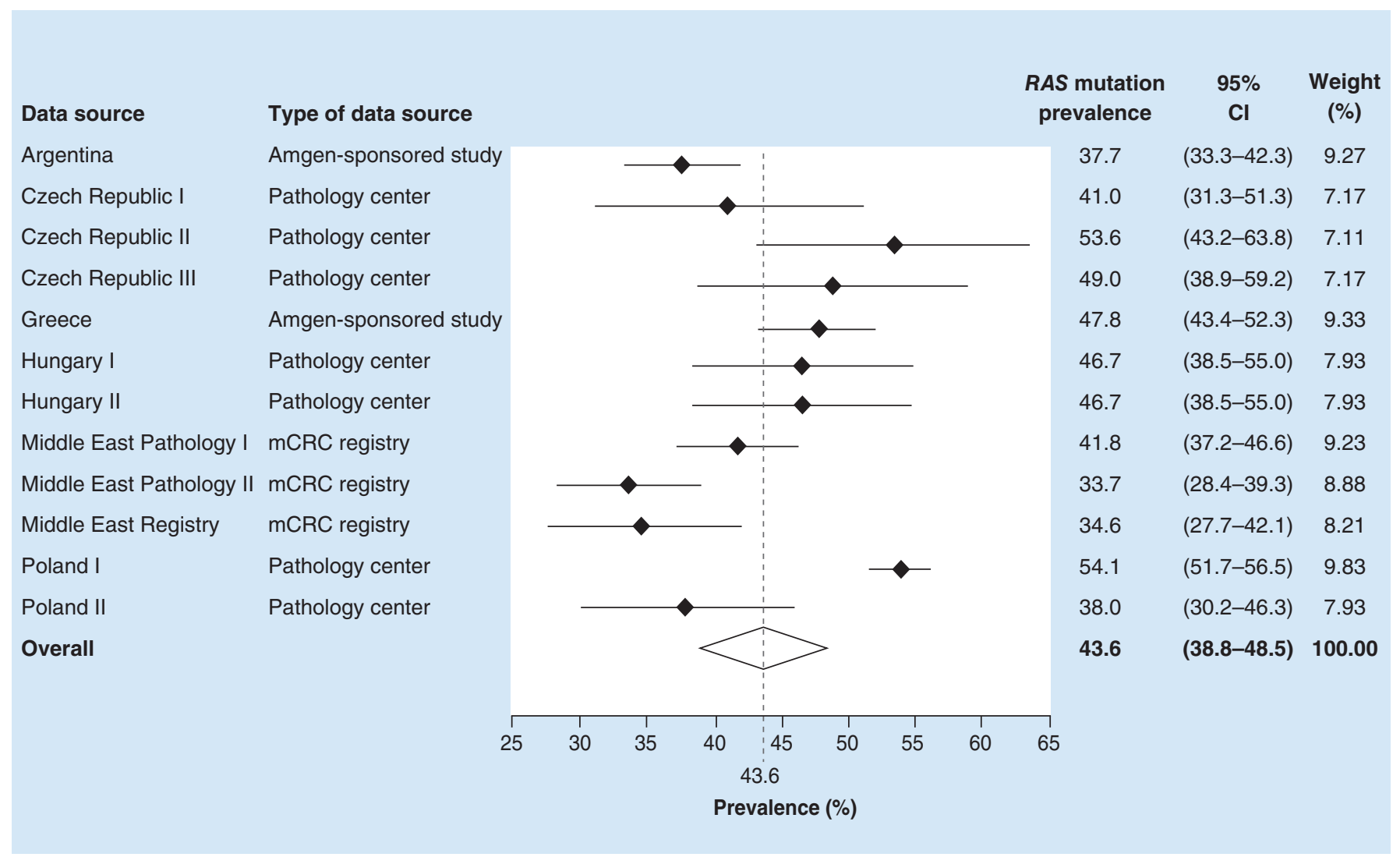

Figure 1. Overall RAS mutation prevalence. Forest plot showing biomarker prevalence by data source. mCRC: Metastatic colorectal cancer.

\section{RAS prevalence by patient characteristics}

Pooled $R A S$ mutation prevalence estimates varied according to certain demographics and clinical variables. Although none of the differences were statistically significant, numerically greater $R A S$ mutation prevalence estimates were observed

in: patients who were aged $\geq 70$ years compared with younger patients; tumor samples isolated from metastatic rather than primary tumor sites; and tumor samples with a ratio of $\geq 20 \%$ neoplastic cells to normal cells in the tumor sample versus $<20 \%$ (Table 2 ).

There were no differences in prevalence estimates for any of the other variables assessed including: patient gender; the tissue from which the sample DNA was isolated (left colon, right colon or rectum); whether or not there was evidence of liver metastases at the time the sample was taken; if there were synchronous or metachronous metastases; or tumor stage (Table 2).

\section{$B R A F$ mutation prevalence}

The overall pooled $B R A F$ mutation prevalence was estimated as $5.8 \%$ (95\% CI: 2.7-9.9\%) across the six data sources. The BRAF mutation prevalence estimates varied from 2.7\% (95\% CI: $0.7-6.7 \%$ ) in one of the Poland pathology datasets to $14.3 \%$ (95\% CI: 6.4-26.2\%) in one of the Czech Republic pathology datasets (Figure 2).

\section{Non-KRAS exon 2 mutation prevalence in KRAS exon 2 wild-type samples}

The overall pooled non-KRAS exon 2 mutation prevalence, in $K R A S$ exon 2 wild-type samples, was estimated as 14.2\% (95\% CI: 10.4-18.4\%). The prevalence estimates varied from $8.6 \%$ (95\% CI: $5.3-13.2 \%$ ) in one of the Middle Eastern mCRC pathology datasets to $22.3 \%$ (95\% CI: $14.7-31.6 \%)$ in a Hungarian pathology center (Figure 3). 


\begin{tabular}{|c|c|c|c|c|}
\hline Variable & Subgroups & $\begin{array}{l}\text { Pooled RAS prevalence } \\
\text { estimate }(\%)\end{array}$ & $95 \% \mathrm{Cl}$ & $\begin{array}{l}\text { Number of data sources included } \\
\text { in analysis }\end{array}$ \\
\hline $\begin{array}{l}\text { Overall RAS mutation } \\
\text { prevalence }\end{array}$ & & 43.6 & $38.8-48.5$ & 12 \\
\hline \multirow[t]{2}{*}{ Age (years) } & $18-49$ & 42.9 & $29.7-56.5$ & 6 \\
\hline & $50-69$ & 40.9 & $33.9-48.2$ & 12 \\
\hline \multirow[t]{2}{*}{ Gender } & Female & 43.3 & $36.8-49.9$ & 10 \\
\hline & Male & 43.8 & $39.0-48.6$ & 12 \\
\hline \multirow{2}{*}{$\begin{array}{l}\text { Tissues from which DNA was } \\
\text { isolated }\end{array}$} & Left colon & 42.7 & $37.8-47.6$ & 9 \\
\hline & Right colon & 45.5 & $35.5-55.7$ & 9 \\
\hline \multirow[t]{2}{*}{ Liver metastases $^{\dagger}$} & Liver metastasis & 39.7 & $30.3-49.4$ & 7 \\
\hline & No liver metastasis & 43.2 & $36.5-50.1$ & 6 \\
\hline \multirow[t]{2}{*}{ Metastatic development } & Synchronous & 47.5 & $40.1-55.0$ & 5 \\
\hline & Metachronous & 42.0 & $32.4-51.9$ & 6 \\
\hline \multirow[t]{3}{*}{ Tumor stage $^{\dagger}$} & Stage $1 / I I$ & 44.0 & $33.5-54.7$ & 5 \\
\hline & Stage III & 40.4 & $32.4-48.6$ & 5 \\
\hline & Stage IV & 43.7 & $35.8-51.8$ & 6 \\
\hline \multirow[t]{3}{*}{ Line of treatment ${ }^{\dagger}$} & 1st line & 49.3 & $40.7-57.8$ & 2 \\
\hline & 2nd line & 35.8 & $31.2-40.6$ & 2 \\
\hline & 3rd line or later & 39.2 & $33.4-45.1$ & 2 \\
\hline
\end{tabular}

\section{Table 3. RAS mutation testing turnaround time by data source.}

Data source Number of samples

Number of samples $\leq 5$

Czech Republic I

Czech Republic II

Czech Republic III

Greece

Hungary I

Hungary II

Middle East Pathology I

Middle East Pathology II

Middle East Registry

Poland I

Poland II

Overall

$100 \quad 37(37.0)$

$\leq 5$

$43(43.0)$

$56(56.0)$

$0(0)$

37 (24.7)

$46(30.7)$

262 (58.6)

274 (90.4)

33 (20.9)

1368 (82.2)

27 (18.0)

2183 (57.0)

Turnaround time (working days), n (\%)

\begin{tabular}{|ll|}
\hline $6-10$ & $>10$ \\
$53(53.0)$ & $10(10.0)$ \\
\hline $47(47.0)$ & $10(10.0)$ \\
\hline $39(39.0)$ & $5(5.0)$ \\
\hline $510(100.0)$ & $0(0)$ \\
\hline $113(75.3)$ & $0(0)$ \\
\hline $73(48.7)$ & $31(20.7)$ \\
\hline $185(41.4)$ & $0(0)$ \\
\hline $29(9.6)$ & $0(0)$ \\
\hline $61(38.6)$ & $64(40.5)$ \\
\hline $274(16.5)$ & $22(1.3)$ \\
\hline $71(47.3)$ & $52(34.7)$ \\
\hline $1455(38.0)$ & $194(5.1)$ \\
\hline
\end{tabular}

\section{RAS testing turnaround time}

Eleven data sources reported $R A S$ testing turnaround times for 3832 samples. Nearly all (94.9\%) of the RAS mutation test results were reported to the requesting physician within 10 working days of the test being requested, with $57.0 \%$ being reported in $1-5$ days, $38.0 \%$ in 6-10 days and only $5.1 \%$ in $>10$ days (Table 3 ). 


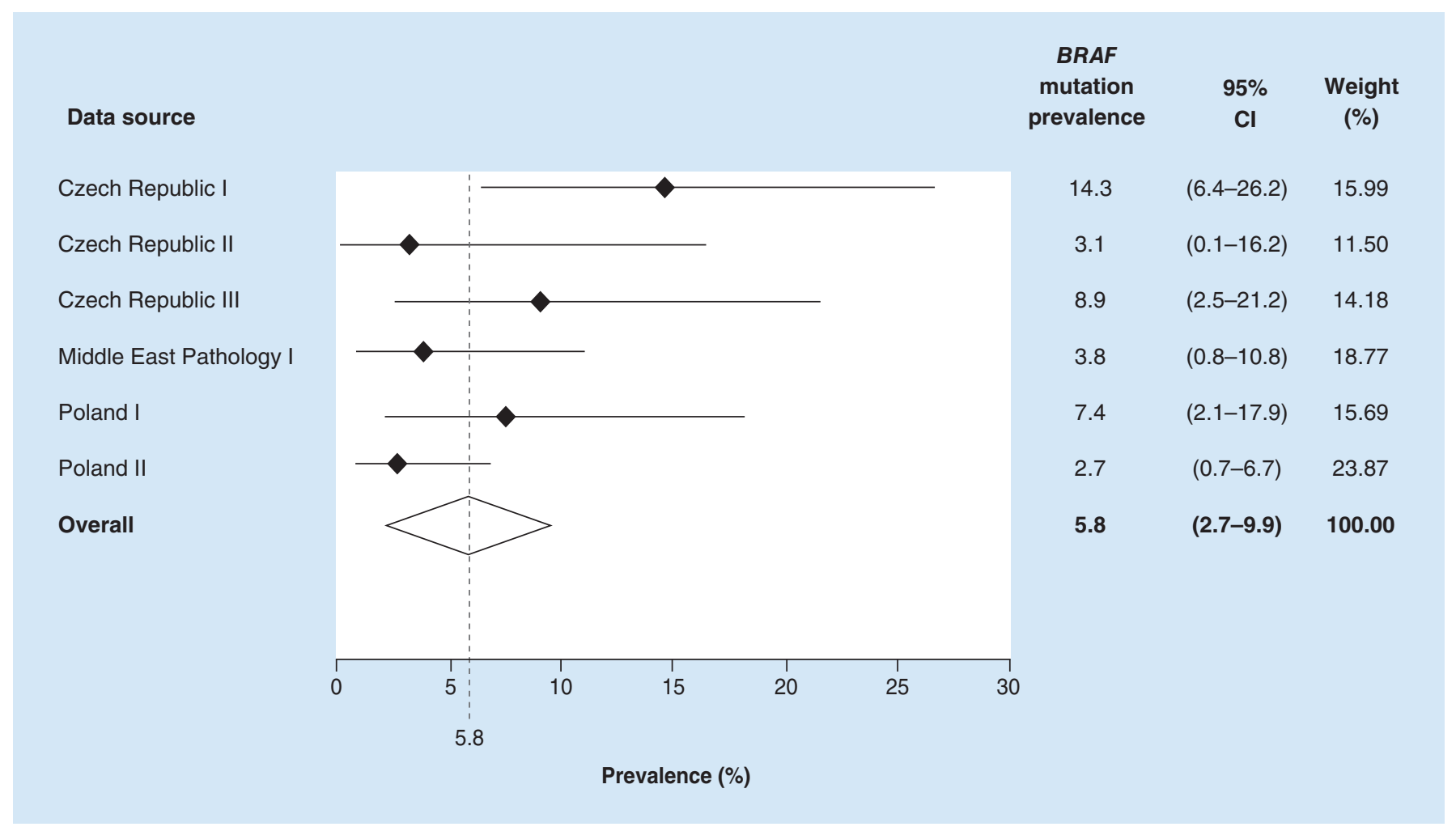

Figure 2. Overall BRAF mutation prevalence.

\section{Discussion}

To our knowledge, this is the largest research project to date to carry out a pooled analysis of RAS mutation prevalence specifically using real-world data. The overall pooled $R A S$ mutation prevalence across all data sources included in the study was estimated to be $43.6 \%$ (95\% CI: 38.8-48.5\%). However, the $R A S$ mutation prevalence estimates for individual data sources included in this analysis varied considerably. Elucidating the reasons for this variability was beyond the scope of the current study but may relate to the ethnic diversity of the patient cohorts in the various countries/regions from which the data were derived. Numerous epidemiological data show major geographical variation with significantly higher risk of CRC in affluent societies and prospective cohort data have linked dietary habits and lifestyle factors to CRC, which may contribute to RAS mutation heterogeneity [12]. The largely lower rate of CRC seen in developing countries of Asia and Africa compared with western countries may also relate to the fact that studies on the prevalence and raw data are very blurred and not definite [13]. The current study highlights the need for further research regarding the reasons for geographical variation in $R A S$ mutation prevalence.

The overall prevalence estimate from this study is somewhat different to estimates reported from past clinical trials. A retrospective analysis of pooled data from five randomized controlled studies (RCTs) of panitumumab estimated the overall unadjusted $R A S$ mutation prevalence in patients with mCRC as 55.9\% (95\% CI: 53.9$57.9 \%)$; however, the prevalence estimate presented here is more consistent with the results of another recent real-world study, which found the overall $R A S$ mutation prevalence reported by European pathology laboratories to be $48.5 \%$ (95\% CI: 46.4-50.6\%) [11,12]. This suggests that the actual prevalence in real-world clinical practice may be lower, meaning that more patients are potential candidates for anti-EGFR treatment than might have been previously thought. Potential reasons for this apparent imbalance may relate to the necessary application of restrictive RCT selection criteria to determine which patients are eligible to participate in controlled trials and which may result in study cohorts not representing the wider patient population observed in routine clinical practice. The wider availability of next-generation sequencing for patient selection or characterization in an RCT setting may also have driven the apparent bias toward an overestimate of the prevalence of RAS mutation among patients with mCRC. This approach may have a lower detection limit than standard laboratory tests for the detection of 


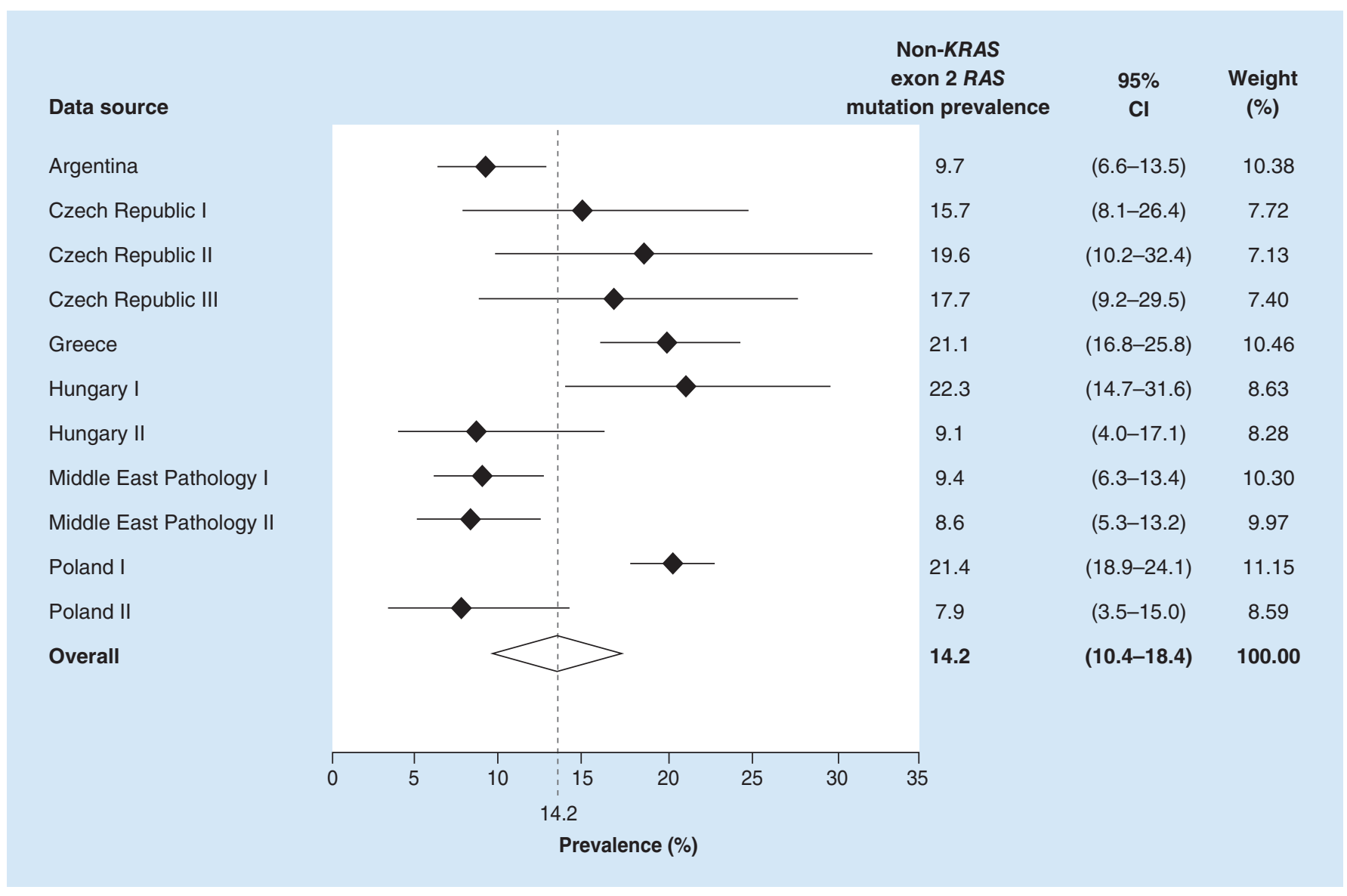

Figure 3. Overall non-KRAS exon 2 RAS mutation prevalence, in patients with a confirmed wild-type KRAS exon 2 status.

low copy number mutant alleles [13]. Alternatively, standard laboratory tests may be less sensitive than those used during RCTs.

The overall pooled $B R A F$ mutation prevalence of $5.8 \%$ was similar to findings from previous RCTs, where $B R A F$ mutation prevalence was estimated as 8.1\% (95\% CI: 6.7-9.6\%) [11]. Similarly, the prevalence of other $R A S$ mutations (KRAS exons 3, 4 and NRAS exons 2, 3, 4) in samples with a confirmed KRAS exon 2 wild-type status was numerically lower than the estimate reported by a recent meta-analysis of both panitumumab and cetuximab RCTs, which found that $19.9 \%$ (95\% CI: 16.7-23.4\%) of samples had a non-KRAS exon 2 mutation. However, the results are within the margin of error of each other [14].

The $R A S$ testing turnaround time was no greater than 10 working days for nearly all of the samples (94.9\%) included in the different data sources. This is marginally higher than reported in a survey of pathology centers in Europe, carried out at the end of 2014, which estimated that for $90.8 \%$ of the laboratories the turnaround time was less than 10 working days [12]. However, this estimate only includes the time between a laboratory receiving the sample and reporting back to the physician. Hence, it is likely that the time from a physician making the request to receiving the sample result is actually longer.

Data collection methodology among pathology laboratories has previously been shown to be highly variable, with a number of different DNA extraction and mutation testing methods being used [15]. This is important to note given the recognized differences in sensitivity among different genotype-ascertainment methodologies [16,17]. In addition, potential quality issues of sample preparation could explain some of the variability. One of the limitations of this research project is that it did not collect data relating to the quality of the DNA samples tested or ascertain if the pathology laboratories involved in the testing were participating in an external quality assurance scheme. This highlights the importance of external quality assurance schemes, particularly given the number of $R A S$ testing methods that have not yet been validated and the lack of standardization among pathology laboratories $[16,18,19]$. 
Blood-based RAS mutation analysis is now available in many countries and may address some of these concerns if used in future clinical trials [20]. Although institutes may or may not be accredited by an independent body, it is a requirement of the European Medicines Authority that $R A S$ mutational status should be determined by an experienced laboratory using validated test methods.

Additionally, the prevalence estimates reported here should be interpreted with caution as the data sources are highly heterogeneous. The heterogeneity of the data could be explained by differences in data collection methodology and the original purposes of the data collection. Differences between eligibility criteria, patient characteristics and study periods may have contributed to $R A S$ prevalence differences. Some of the included samples may have been initially tested for KRAS exon 2 status with testing for the remaining RAS exons (KRAS exons 3, 4 and NRAS exons 2, 3, 4) conducted at a later time point. In practice, samples were likely to have been collected at different time points (e.g., time of surgery), including some which may have been collected prior to metastatic diagnosis (most likely stage III CRC).

\section{Conclusion}

This pooled analysis of $R A S$ mutation prevalence estimates from real-world data sources found the overall $R A S$ mutation prevalence among patients with $\mathrm{mCRC}$ to be somewhat lower than reported in clinical trials.

\section{Future perspective}

New knowledge of biomarkers involved in mCRC will impact the future screening, diagnosis and treatment of the disease. This may lead to new anticancer agents that are selective for certain subgroups of patients and to more precise use of the currently available agents. This will increase treatment options available to clinicians and may lead to tailored individualistic approaches, decreasing treatment cost and the adverse effects related to $\mathrm{mAb}$ therapy.

Authors' contributions

All authors were involved in, and contributed to, the drafting and critical review of this manuscript.

\section{Acknowledgements}

The authors would like to thank and acknowledge the following people for their contributions to this article: A Bray (Amgen Ltd) for study management support; S Jani and the Adelphi Research team for data management; E Benes-Javor (Adelphi Research) for providing statistical input; D Spanopoulos (Amgen Ltd) for data management support; and Adelphi Communications for editorial assistance and support (funded by Amgen Ltd).

\section{Financial \& competing interests disclosure}

This research project was funded by Amgen Ltd. Amgen was also involved in the study design, data collection and analysis, decision to publish and preparation of the manuscript. Individual conflict of interest disclosures are given below. $G$ Kafatos is a compensated employee of Amgen Ltd as an Observational Research Senior Manager and a stockholder in Amgen Ltd. D Niepel is a compensated employee of Amgen $\mathrm{GmbH}$ as a Medical Director and a stockholder in Amgen GmbH. K Lowe is a compensated employee of Amgen Inc. as an Epidemiology Senior Manager and a stockholder in Amgen Inc. T Garawin is a compensated employee of Amgen Inc. as the Head of Oncology (Middle East) and a stockholder in Amgen Inc. Z Traugottová is a compensated employee of Amgen s.r.o. as a Senior Medical Advisor and a stockholder in Amgen Ltd. A Bilalis is a compensated employee of Amgen Hellas EPE as a Senior Medical Advisor. E Molnar is a compensated employee of Amgen Kft as a Senior Medical Advisor. J Timar has received honoraria from Amgen, Boehringer Ingelheim, Eli Lilly, Merck Serono, Pfizer and Roche. P Fabian has received consultation fees from Amgen and Pfizer, and honoraria from Amgen, AstraZeneca, Merck Serono, Pfizer and Roche. J Han van Krieken has received research funding from Amgen and Merck Serono, speaker fees from Amgen, Merck Serono, Agilent (DAKO) and Roche and consulting fees from Milestone Pharmaceuticals and Sakura. J Trojan has received speaker fees from Amgen and Bayer, and consulting fees from Bayer, Eli Lilly and Merck Serono. S Murray has received consulting fees from Amgen. P Wójcik is a Co-owner and Board member of Oncogene Diagnostics sp. z o.o. S Jenkins-Anderson, H Westhead, E Toth, N Gouvas, G Papaxoinis, N Mokhtar, H Vosmikova, A Skalova, A Tysarowski and M Barugel have no competing interests to declare. The authors have no other relevant affiliations or financial involvement with any organization or entity with a financial interest in or financial conflict with the subject matter or materials discussed in the manuscript apart from those disclosed.

Amgen Ltd. provided funding for medical writing assistance and journal publication fees. 
Ethical conduct of research

The authors state that they have obtained appropriate institutional review board approval or have followed the principles outlined in the Declaration of Helsinki for all human or animal experimental investigations. In addition, for investigations involving human subjects, informed consent has been obtained from the participants involved.

\section{Summary points}

- RAS mutation status is an important predictive biomarker of successful outcome for patients with metastatic colorectal cancer (mCRC) treated with anti-EGFR therapies such as cetuximab and panitumumab.

- Therefore, in many countries, a confirmed wild-type RAS (exons 2, 3, 4 of KRAS and NRAS) tumor status is a requirement for prescribing anti-EGFR treatment to patients with $\mathrm{mCRC}$.

- Our currently limited understanding of RAS mutation prevalence in $\mathrm{mCRC}$ is largely derived from clinical trials; this research project aims to estimate the prevalence on RAS mutations based on real-world data sources.

- Aggregate patient data were collected from a range of geographically diverse real-world sources, by means of an online standardized form, and included in an overall meta-analysis.

- The overall pooled RAS mutation prevalence for 4431 tumor samples across 12 data sources was estimated to be $43.6 \%$.

- RAS mutation prevalence varied between data sources, ranging from low estimates of $33.7 \%$ in a Middle Eastern pathology dataset and $34.6 \%$ in a Middle Eastern mCRC registry to the highest estimates of $53.6 \%$ and $54.1 \%$ in two European pathology centers, in the Czech Republic and Poland, respectively.

- The overall pooled RAS mutation prevalence was lower than previously reported in a pooled analysis of randomized controlled studies of anti-EGFR treatment; however, it was more consistent with the results of a similar, albeit smaller, real-world study.

- Therefore, the actual real-world prevalence may be lower than reported in randomized controlled studies, meaning that more patients are potential candidates for anti-EGFR treatment than might have been previously thought.

\section{References}

Papers of special note have been highlighted as: $\bullet$ of interest; $\bullet \bullet$ of considerable interest

1 Torre LA, Siegel RL, Ward EM, Jemal A. Global cancer incidence and mortality rates and trends-an update. Cancer Epidemiol. Biomarkers Prev. 25, 16-27 (2016).

2 Ferlay J, Soerjomataram I, Dikshit R et al. Cancer incidence and mortality worldwide: sources, methods and major patterns in GLOBOCAN 2012. Int. J. Cancer 136, E359-E386 (2015).

3 van Cutsem E, Cervantes A, Nordlinger B, Arnold D. ESMO Guidelines Working Group. Collaborators (20), Ann. Oncol. 25(Suppl. 3), iii1-iii9 (2014).

- Treatment guidelines for managing patients with metastatic colorectal cancer (mCRC), including recommendations for expanded $R A S$ mutation status testing prior to initiation of anti-EGFR therapy.

4 Tay RY, Wong R, Hawkes EA. Treatment of metastatic colorectal cancer: focus on panitumumab. Cancer Manag. Res. 7, 189-198 (2015).

5 Moorcraft SY, Smyth EC, Cunningham D. The role of personalized medicine in metastatic colorectal cancer: an evolving landscape. Therap. Adv. Gastroenterol. 6, 381-395 (2013).

6 Douillard JY, Oliner KS, Siena S et al. Panitumumab-FOLFOX4 treatment and RAS mutations in colorectal cancer. N. Engl. J. Med. 369, 1023-1034 (2013).

- $\quad$ Retrospective analysis of the PRIME trial, which demonstrated that anti-EGFR treatment of tumors with $R A S$ mutations outside of $K R A S$ exon 2 may lead to detrimental effects on survival outcomes.

7 Schwartzberg LS, Rivera F, Karthaus M et al. PEAK: a randomized, multicenter phase II study of panitumumab plus modified fluorouracil, leucovorin, and oxaliplatin (mFOLFOX6) or bevacizumab plus mFOLFOX6 in patients with previously untreated, unresectable, wild-type KRAS exon 2 metastatic colorectal cancer. J. Clin. Oncol. 32, 2240-2247 (2014).

8 Heinemann V, von Weikersthal LF, Decker T et al. FOLFIRI plus cetuximab versus FOLFIRI plus bevacizumab as first-line treatment for patients with metastatic colorectal cancer (FIRE-3): a randomised, open-label, phase 3 trial. Lancet Oncol. 15, 1065-1075 (2014).

9 Seymour MT, Brown SR, Middleton G et al. Panitumumab and irinotecan versus irinotecan alone for patients with KRAS wild-type, fluorouracil-resistant advanced colorectal cancer (PICCOLO): a prospectively stratified randomised trial. Lancet Oncol. 14, 749-759 (2013).

10 Peeters M, Douillard JY, van Cutsem E et al. Mutant KRAS codon 12 and 13 alleles in patients with metastatic colorectal cancer: assessment as prognostic and predictive biomarkers of response to panitumumab. J. Clin. Oncol. 31, 759-765 (2013). 
11 Peeters M, Kafatos G, Taylor A et al. Prevalence of $R A S$ mutations and individual variation patterns among patients with metastatic colorectal cancer: a pooled analysis of randomised controlled trials. Eur. J. Cancer 51, 1704-1713 (2015).

-• Meta-analysis of five randomized controlled studies of panitumumab in mCRC reporting RAS mutation prevalence estimates from 3196 mCRC patients across 36 countries.

12 Boleij A, Tack V, Taylor A et al. RAS testing practices and $R A S$ mutation prevalence amongst mCRC patients: results from a Europe-wide survey of pathology centres. BMC Cancer 16, 825 (2016) (In Press).

- Survey of pathology laboratories in Europe reporting real-world $R A S$ mutation prevalence rates consistent with the estimates generated by this meta-analysis.

13 Peeters M, Oliner KS, Parker A et al. Massively parallel tumor multigene sequencing to evaluate response to panitumumab in a randomized phase III study of metastatic colorectal cancer. Clin. Cancer Res. 19, 1902-1912 (2013).

14 Sorich MJ, Wiese MD, Rowland A et al. Extended RAS mutations and anti-EGFR monoclonal antibody survival benefit in metastatic colorectal cancer: a meta-analysis of randomized, controlled trials. Ann. Oncol. 26, 13-21 (2015).

- Meta-analysis of nine randomized controlled studies of anti-EGFR therapies, which confirmed the predictive value of expanded $R A S$ mutation testing (beyond KRAS exon 2) in determining likely treatment outcome in mCRC.

15 Boleij A, Tops BB, Rombout PD et al. RAS testing in metastatic colorectal cancer: excellent reproducibility amongst 17 Dutch pathology centers. Oncotarget 6, 15681-15689 (2015).

16 Wong NA, Gonzalez D, Salto-Tellez M et al. RAS testing of colorectal carcinoma - a guidance document from the Association of Clinical Pathologists Molecular Pathology and Diagnostics Group. J. Clin. Pathol. 67, 751-757 (2014).

17 Malapelle U, Carlomagno C, de Luca C et al. KRAS testing in metastatic colorectal carcinoma: challenges, controversies, breakthroughs and beyond. J. Clin. Pathol. 67, 1-9 (2014).

18 van Krieken JH, Jung A, Kirchner T et al. KRAS mutation testing for predicting response to anti-EGFR therapy for colorectal carcinoma: proposal for an European quality assurance program. Virchows Arch. 453, 417-431 (2008).

- Initial guideline recommendations and a proposal for a European quality assurance program to help ensure accuracy and proficiency in KRAS mutation testing.

19 Tack V, Ligtenberg MJ, Tembuyser L et al. External quality assessment unravels interlaboratory differences in quality of $R A S$ testing for anti-EGFR therapy in colorectal cancer. Oncologist 20, 257-262 (2015).

$20 \mathrm{Li} \mathrm{Y,} \mathrm{Fu} \mathrm{XH,} \mathrm{Yuan} \mathrm{JQ} \mathrm{et} \mathrm{al.} \mathrm{Colorectal} \mathrm{cancer:} \mathrm{using} \mathrm{blood} \mathrm{samples} \mathrm{and} \mathrm{tumor} \mathrm{tissue} \mathrm{to} \mathrm{detect} \mathrm{K-ras} \mathrm{mutations.} \mathrm{Expert} \mathrm{Rev.} \mathrm{Anticancer}$ Ther. 15, 715-725 (2015). 